\title{
Estudo fitoquímico biomonitorado pelos ensaios de toxicidade frente à Artemia salina e de atividade antiplasmódica do caule de aninga (Montrichardia linifera)
}

Cristine Bastos do AMARANTE ${ }^{1}$, Adolfo Henrique MÜLLER², Marinete Marins PÓVOA³, Maria Fâni DOLABELA ${ }^{4}$

\section{RESUMO}

Montrichardia linifera é uma planta aquática de amplo uso na medicina tradicional amazônica. Entretanto, muito pouco se conhece sobre a sua composição química, e sua atividade biológica ainda não foi comprovada. Na busca de substância(s) biologicamente ativa(s), este trabalho realizou um estudo fitoquímico biomonitorado no qual foram testados os extratos hexânico e etanólico obtidos do caule desta espécie, dos quais apenas o extrato etanólico foi selecionado para o fracionamento cromatográfico por ter apresentado toxicidade contra a Artemia salina e atividade contra o Plasmodium falciparum, parasita causador da malária. As atividades biológicas concentraram-se na fração diclorometânica que apresentou alta toxicidade contra A. salina $\left(\mathrm{DL}_{50}<31 \mu \mathrm{g} \mathrm{mL}^{-1}\right)$ e alta atividade antiplasmódica $\left(\mathrm{IC}_{50}<10 \mu \mathrm{g} \mathrm{mL}^{-1}\right)$, mostrando promissora atividade antimalárica. Desta fração, o composto aromático $p$-hidroxibenzaldeído foi isolado pela primeira vez nesta planta.

PALAVRAS-CHAVE: Montrichardia linifera, toxicidade contra Artemia salina, atividade antiplasmódica.

\section{Phytochemical study bioassay-guided by tests of toxicity on Artemia salina and antiplasmodial activity from stem of aninga (Montrichardia linifera)}

\begin{abstract}
Montrichardia linifera is an aquatic plant widely use in Amazon folkmedicine. However, very little is known about the chemical composition and biological activity. In search of biologically active (s) substance (s) phytochemical bioassay-guided study was conducted evaluating hexane extract and ethanol extract obtained from stems of this species. Since only the ethanol extract presented toxicity against Artemia salina and activity against Plasmodium falciparum, this extract was selected for chromatographic fractionation. The biological activities were concentred in dichloromethane fraction which showed high toxicity against $A$. saline $\left(\mathrm{LD}_{50}<\mu \mathrm{g} \mathrm{mL}^{-1}\right)$ and high antiplasmodial activity $\left(\mathrm{IC}_{50}<10 \mu \mathrm{g} \mathrm{mL}^{-1}\right)$, showing promising antimalarial activity. Of this fraction, the aromatic compound p-hydroxybenzaldehyde was isolated for the first time in this plant.
\end{abstract}

KEYWORDS: Montrichardia linifera, toxicity on Artemia salina, antiplasmodial activity.

\footnotetext{
${ }_{1}$ Museu Paraense Emílio Goeldi - MPEG. cbamarante@museu-goeldi.br

${ }^{2}$ Centro Universitário do Pará - CESUPA. muller@cesupa.br

3 Instituto Evandro Chagas - IEC. marinetepovoa@iec.pa.gov.br

${ }^{4}$ Universidade Federal do Pará - UFPA. fanidolabela@gmail.com
} 
O Brasil possui a maior diversidade genética em espécies de plantas no mundo, porém, menos de $10 \%$ foram avaliadas quanto as suas características biológicas e, menos de 5\% foram submetidas a estudos fitoquímicos detalhados. Apesar de um recente aumento de pesquisas nesta área, as plantas ainda constituem uma fonte relativamente subutilizada e, potencialmente, muito valiosa para descoberta de novas substâncias biologicamente ativas (Luna et al. 2005).

O teste de toxicidade contra a Artemia salina (TAS) é um ensaio biológico considerado como uma das ferramentas mais utilizadas para a avaliaçấo preliminar de toxicidade. Desta forma, A. salina tem sido usada como um organismo alvo para detectar compostos bioativos em extratos de plantas (Alves et al. 2000), a toxicidade para este crustáceo tem demonstrado uma boa correlação com a atividade citotóxica contra tumores humanos (McLaughlin et al. 1991) e atividade contra o Trypanosoma cruzi (Alves et al. 2000; Zani et al. 1995), protozoário causador da doença de Chagas. Em geral, extratos de plantas e derivados com alta toxicidade contra a A. salina sugerem alto potencial para atividades biológicas, sendo, portanto, muito útil a utilizaçấo deste bioensaio, no direcionamento de estudos fitoquímicos, na busca de substâncias bioativas.

A Montrichardia linifera (Arruda) Schott, família Araceae, popularmente conhecida como 'aninga', é uma macrófita aquática que forma grandes populaçôes clonais às margens dos rios e igarapés da Amazônia. Os ribeirinhos consideram esta planta venenosa porque sua seiva causa queimaduras na pele e em contato com os olhos pode causar cegueira (Amarante et al. 2009). Entretanto, paradoxalmente, ela é amplamente usada na medicina tradicional amazônica, principalmente devido à propriedade cicatrizante da seiva e do suco desta planta, que já vem sendo mencionada na literatura desde o século 19 para o tratamento de feridas e úlceras (D’Oliveira 1854; Moreira 1862; Castro 1878 apud Fenner et al. 2006), levando à hipótese que de que esta espécie possa conter substâncias biologicamente ativas. Porém, ainda muito pouco se conhece sobre a sua composição química e atividades biológicas que, eventualmente, poderão ser úteis também contra infecçôes humanas causadas por parasitas, que são sérios problemas em países tropicais e subtropicais em desenvolvimento apesar da descoberta de novos medicamentos anti-protozoários (MooPuc et al. 2008). Entre os compostos antimaláricos isolados de plantas, a artemisinina (Figura 1) é a descoberta mais recente e mais importante da atualidade (Osorio et al. 2006; Vikas et al. 2000). Há no entanto, a necessidade da descoberta de novas drogas antimaláricas. Neste sentido, o objetivo deste trabalho foi pesquisar a espécie $M$. linifera utilizando extratos e fraçōes obtidos do caule para a realização de prospecção fitoquímica preliminar, guiada pelos ensaios biológicos de toxicidade frente à Artemia salina e atividade antiplasmódica, para se investigar

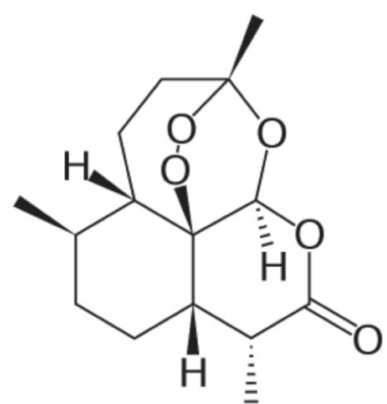

Figura 1 - Estrutura química da artemisinina

o potencial desta planta em apresentar substância(s) ativa(s) contra o Plasmodium falciparum, parasita causador da malária.

O extrato hexânico e o etanólico foram obtidos de caules de $M$. linifera coletados na Universidade Federal do Pará, Belém-PA, à margem direita do Rio Guamá $\left(01^{\circ} 28^{\prime} 41,3^{\prime \prime} \mathrm{S}\right.$

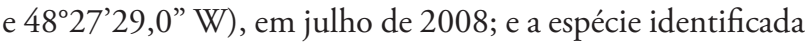
pela Dra Alba Lúcia F. A. Lins (Coordenaçấo de Botânica do Museu Paraense Emílio Goeldi), exsicata MG 188906. Os extratos foram submetidos aos bioensaios de toxicidade frente à Artemia salina e atividade antiplasmódica para seleção do material a ser estudado. $\mathrm{O}$ extrato etanólico apresentou alta toxicidade para Artemia salina e moderada atividade antiplasmódica, sendo selecionado para o fracionamento. Amostra de 53,6 g deste extrato foi submetida à cromatografia de coluna aberta de Silica gel $60(230$ - 400 mesh, ASTM. Merck) e eluída com hexano, diclorometano, acetato de etila e metanol. As quatro fraçóes obtidas também foram biomonitoradas e a amostra selecionada submetida a análises de Infravermelho (espectrômetro Thermo Scientific, modelo Nicolet IR100) e de ressonância magnética nuclear de hidrogênio e carbono ( $\mathrm{RMN}{ }^{1} \mathrm{H} \mathrm{e}{ }^{13} \mathrm{C}$ ), obtidos utilizando um espectrômetro Varian, modelo Mercury-300 (300 MHz para ${ }^{1} \mathrm{H}$ e $75 \mathrm{MHz}$ para ${ }^{13} \mathrm{C}$ ) e usando o solvente DMSO-d ${ }_{6}$ como padrão interno.

A avaliação da toxicidade dos extratos e fraçôes foi realizada através do bioensaio com Artemia salina Leach, de acordo com McLaughlin et al. (1993), testadas em cinco concentraçôes diferentes (500; 250; 125; 62,50 e 31,25 $\left.\mathrm{\mu g} \mathrm{mL}^{-1}\right)$, dissolvidas em DMSO. Para o cálculo da $\mathrm{DL}_{50}$ foi utilizado o método GraphPadPrism 5, e considerou-se baixa toxicidade quando a dose letal $50 \%\left(\mathrm{DL}_{50}\right)$ foi superior a $500 \mu \mathrm{g} \mathrm{mL}^{-1}$; moderada para $\mathrm{DL}_{50}$ entre 100 a $500 \mu \mathrm{g} \mathrm{mL}^{-1}$ e muito tóxico quando a $\mathrm{DL}_{50}$ foi inferior $100 \mu \mathrm{g} \mathrm{mL}^{-1}$.

Para a avaliação da atividade antiplasmódica, o cultivo do Plasmodium falciparum Dd2, resistente a cloroquina e sulfadoxina, foi realizado de acordo com Trager e Jensen (1976). A metodologia utilizada neste ensaio foi descrita por Rieckman et al. (1978) e modificada por Carvalho (1990). 
As amostras foram solubilizadas em RPMI e 0,025\% (v/v) de dimetilssulfóxido, sendo utilizadas as seguintes concentraçôes: 3,$125 ; 6,25 ; 12,5 ; 25 ; 50$ e $100 \mu \mathrm{gL}^{-1}$. Como controle positivo foi utilizado cloroquina em diferentes concentraçóes. Após 72 horas, foram confeccionados os esfregaços, determinando-se a parasitemia percentual, a partir da qual calculou-se a $\mathrm{CI}_{50}$ utilizando-se o método Graph Pad Prism 4,0 . Os resultados foram expressos baseados na média de dois experimentos, considerou-se alto potencial antimalárico quando a $\mathrm{CI}_{50}$ foi inferior a $10 \mu \mathrm{g} \mathrm{mL}^{-1}$, potencial moderado para $\mathrm{CI}_{50}$ entre 10 e $100 \mu \mathrm{g} \mathrm{mL}^{-1} \mathrm{e}$ baixo potencial quando a $\mathrm{CI}_{50}$ foi superior a $100 \mu \mathrm{g} \mathrm{mL}$.

Os dados dos rendimentos (em \%), da toxicidade $A$. salina (TAS), expressa em Dose Letal 50\%, e da atividade antiplasmódica contra o clone de P. falciparum $\mathrm{Dd} 2$, resistente a cloroquina e sulfadoxina, expressa em Concentração Inibitória $50 \%$, nas seis amostras ensaiadas, estáo mostrados na Tabela 1. Quatro das seis amostras testadas apresentaram alta toxicidade $\left(\mathrm{DL}_{50}<100 \mu \mathrm{g} \mathrm{mL} \mathrm{m}^{-1}\right)$ contra $A$. salina. Com relação à atividade antiplasmódica, duas amostras apresentaram alto potencial antimalárico $\left(\mathrm{CI}_{50}<10 \mu \mathrm{g} \mathrm{mL}^{-1}\right)$ e outras duas apresentaram potencial moderado $\left(10<\mathrm{CI}_{50}<100 \mu \mathrm{g} \mathrm{mL} \mathrm{mL}^{-1}\right)$. Entretanto, apenas as fraçóes diclorometânica e acetato de etila apresentaram os mesmos efeitos entre a atividade antiplasmódica e a toxicidade contra a Artemia salina, isto é, a fração diclorometânica apresentou alta toxicidade contra A. salina $\left(\mathrm{DL}_{50}<31 \mu \mathrm{g} \mathrm{mL}^{-1}\right)$ e alto potencial antimalárico $\left(\mathrm{CI}_{50}<10 \mu \mathrm{g} \mathrm{mL} \mathrm{m}^{-1}\right)$ e a fração acetato de etila apresentou atividade moderada em ambos os ensaios $\left(\mathrm{DL}_{50}=155,4 \pm\right.$ $1,6 \mu \mathrm{g} \mathrm{mL}^{-1}$ e $10<\mathrm{CI}_{50}<100 \mu \mathrm{g} \mathrm{mL}^{-1}$, respectivamente). Por ter apresentado as maiores atividades em ambos os ensaios, a fração diclorometânica foi a selecionada para purificação através de cromatografia em placa preparativa e forneceu uma substância química. Os sinais obtidos do espectro de $\mathrm{RMN}{ }^{1} \mathrm{H}$ indicaram a presença de um anel aromático p-substituído, com um dupleto em torno $\mathrm{de}_{\mathrm{H}} 7,75 \mathrm{ppm}(J$
$=8,5 \mathrm{~Hz})$ e outro dupleto em $\mathrm{d}_{\mathrm{H}} 6,93 \mathrm{ppm}(J=8,5 \mathrm{~Hz})$, e correspondem ao acoplamento em orto entre os hidrogênios do anel aromático (Pavia et al. 1996; Silverstein e Webster 2000). Além disso, apresentou um simpleto em campo baixo $\mathrm{d}_{\mathrm{H}} 9,79 \mathrm{ppm}(1 \mathrm{H})$, característico de hidrogênio de aldeído. $\mathrm{O}$ espectro na regiáo do infravermelho desta substância corrobora com estas evidências, pois apresentou um pico em $1712 \mathrm{~cm}^{-1}$, característico do grupo carbonila de aldeídos com insaturaçáo conjugada, como nos derivados do benzaldeído que reduzem a freqüência de absorção da carbonila, passando a ser encontrada na região de $1710-1685 \mathrm{~cm}^{-1}$ (Silverstein et al. 2007). Considerando os resultados espectrais obtidos, propóe-se para o produto a estrutura do $p$-hidroxibenzaldeído (Figura 2). Não existe nenhum dado na literatura relacionado à presença deste composto na Montrichardia linifera, sendo isolado desta planta pela primeira vez. Estudos anteriores já mostraram a atividade antimalárica de $p$-hidroxibenzaldeído, isolado de algas do gênero Laurencia, contra o Plasmodium falciparum (Mendiola-Martínez et al. 2005; Wright et al. 1996; Topcu et al. 2003), corroborando com os resultados obtidos para a alta atividade antiplasmódica encontrada na fração diclorometânica neste trabalho.

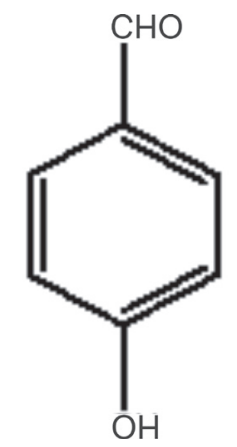

Figura 2 - Estrutura química do $p$-hidroxibenzaldeído isolado do caule de M. linifera

Tabela 1 - Rendimento (\%), avaliação da toxicidade em A. salina $\left(\mathrm{DL}_{50}\right)$ e atividade antiplasmódica $\left(\mathrm{Cl}_{50}\right)$ de extratos e frações obtidos do caule de $M$. linifera.

\begin{tabular}{lcll} 
Amostras & \multicolumn{1}{c}{$\begin{array}{c}\text { Rendimento } \\
(\%)\end{array}$} & \multicolumn{1}{c}{$\begin{array}{c}\text { TAS } \\
\mathrm{DL}_{50}\left(\mu \mathrm{g} \mathrm{mL}{ }^{-1}\right) \pm \mathrm{SD}\end{array}$} & $\begin{array}{c}\text { Atividade antiplasmódica } \\
\mathrm{Cl}_{50}{ }^{*}\left(\mu \mathrm{mL}^{-1}\right)\end{array}$ \\
\hline Extrato hexânico & 0,28 & $<31$ (alta) & $\mathrm{Cl}_{50}>100$ (baixa) \\
Extrato etanólico & 2,89 & $60,35 \pm 1,15$ (alta) & $10<\mathrm{Cl}_{50}<100$ (moderada) \\
Fração hexânica & 0,28 & $<31$ (alta) & $\mathrm{Cl}_{50}>100$ (baixa) \\
Fração diclorometânica & 4,06 & $<31$ (alta) & $\mathrm{Cl}_{50}<10$ (alta) \\
Fração acetato de etila & 13,83 & $155,4 \pm 1,6$ (moderada) & $10<\mathrm{Cl}_{50}<100$ (moderada) \\
Fração metanólica & 53,48 & $>500$ (baixa) & $\mathrm{Cl}_{50}<10$ (alta) \\
\hline
\end{tabular}

TAS (Toxicidade em Artemia salina); $\mathrm{DL}_{50}$ (Dose Letal para 50\% da população em teste); $\mathrm{Cl}_{50}$ (Concentração Inibitória de 50\% da população em teste). *Para comparação: $\mathrm{Cl}_{50}$ para artemisinina (Figura 1) $=10^{-3} \mu \mathrm{g} \mathrm{mL}^{-1}$. 


\section{AGRADECIMENTOS}

Ao Prof. Dr. José Roberto Zamian da Universidade Federal do Pará pelas análises de Infravermelho, à Dra Alba Lúcia Ferreira de Almeida Lins do Museu Paraense Emílio Goeldi, pela identificação do material botânico e à Fundaçãoo de Amparo à Pesquisa do Estado do Pará (FAPESPA) pelo suporte financeiro.

\section{BIBLIOGRAFIA CITADA}

Alves, T M.A.; Silva, A.F.; Brandão, M.; Grandi, T.S.M.; Smânia, E.F.A.; Smânia Jr., A.; Zani, C.L. 2000. Biological screening of Brazilian medicinal plants. Memórias do Instituto Oswaldo Cruz, 95: 367-373.

Amarante, C.B.; Silva, J.C.F.; Solano, F.A.R.; Nascimento, L.D.; Moraes, L.G.; Silva, G.F.; Uno, W.S. 2009. Spectrometric study of the leaves of Aninga (Montrichardia linifera) collected from the Guama River, Campus of UFPA, Belém-PA. A contribution to the chemical study of the Araceae family. Revista Cientifica da UFPA, 7: 1-19. (In Portuguese, with abstract in English).

Castro, J.M. 1878. Purgatives Brazil's indigenous. Dissertação de Mestrado, Faculdade de Medicina do Rio de Janeiro. 186 pp. (in Portuguese).

Carvalho, L.H. 1990. Experimental chemotherapy with crude extracts from plants and chemically defined compounds. Dissertação de Mestrado, Universidade Federal de Minas Gerais, Belo Horizonte, Minas Gerais. 146 pp. (in Portuguese).

D'Oliveira, H.V. 1854. System of Brazilian vegetable materia medica. Casa de Eduardo \& Henrique Laemmert, Rio de Janeiro, Brasil. 284 pp. (in Portuguese).

Fenner, R.; Betti, A.H.; Mentz, L.A.; Rates, S.M.K. 2006. Plants with potencial antifungal activity employed in Brazilian folk medicine. Revista Brasileira de Ciências Farmacêuticas, 42: 369394. (In Portuguese, with abstract in English).

Luna J.S.; Santos, A.F.; Lima, M.R.F.; Omena, M.C.; Mendonça, F.A.C.; Bieber, L.W.; Sant'Ana, A.E.G. 2005. A study of the larvicidal and molluscicidal activities of some medicinal plants from northeast Brazil. Journal of Ethnopharmacology, 97: 199206.

McLaughlin, J.L.; Chang, C.J.; Smith, D.L. 1991. "Bench-top" bioassays for the discovery of bioactive natural products: an update, p. 383-409. In: Rahman, A. (Org.). Studies in Natural Product Chemistry, $9^{\text {th }}$ ed., Elsevier, Amsterdam.

McLaughlin, J.L.; Chang, C.J.; Smith, D.L. 1993. Simple Bench-Top Bioassays (BS \& PD) for Discovery of Plant Antitumor Compounds - Review of Recent Progress, p. 112-137. In: Kinghorn, A.D.; Balandrini, M.F. (Eds). Human Medicinal Agents from Plants, Oxford University Press, New York, USA.
Mendiola-Martínez, J.; Hernández, H.; Acuña, D.; Esquivel, M.; Lizama, R.S.; Payrol, J.A. 2005. Inhibiting activity of the in vitro growth of Plasmodium falciparum of extracts from algae of genus Laurencia. Revista Cubana de Medicina Tropical, 57: 192-195 (In Spanish, with abstract in English).

Moreira, N.J. 1862. Dictionary of Brazilian medicinal plants. Typographia do Correio Mercantil, Rio de Janeiro, Brasil. 144 pp. (In Portuguese).

Moo-Puc, R.; Robledo, D.; Freile-Pelegrin, Y. 2008. Evaluation of selected tropical seaweeds for in vitro anti-trichomonal activity. Journal of Ethnopharmacology, 120: 92-97.

Osorio, D.E.J.; Montoya P.G.L.; Arango A.G.J. 2006. Alkaloidal natural products with antiprotozoal activity. Vitae, 13: 61-84. (in Spanish, with abstract in English).

Pavia, D.L.; Lampman, G.M.; Kriz, G.S. 1996. Introduction to Spectroscopy. 2 ed. Saunders College Publishing, Filadélfia, USA.

Rieckman, K.H.; Sax, L.J.; Campbell, G.H.; Mrena, J.F. 1978. Drug sensitivity of Plasmodium falciparum. An in vitro microtechnique. The Lancet, 311: 22-23.

Silverstein, R.M; Webster, F.X.; Kiemle, D.J. 2000. Spectrometric identification of organic compounds. 6.ed. LTC, Rio de Janeiro, Brasil. 460 pp. (In Portuguese).

Silverstein, R.M; Webster, F.X. 2007. Spectrometric identification of organic compounds. 7ed. LTC, Rio de Janeiro, Brasil. 490 pp. (In Portuguese).

Trager, W.; Jensen, J.B. 1976. Human malaria parasites in continuous culture. Science, 193: 673-675.

Topcu, G.; Anydoqmus, Z.; Imre, S.; Goren, A.C.; Pezzuto, J.M.; Clement, J.A.; Kingston, D.G. 2003. Brominated sesquiterpenes from the alga Laurencia obtuse. Journal of Natural Products, 66: 1505-1508.

Wright, A.D.; König, M.; Angerhofer, C.K.; Greenidge, P.; Linden, A.; Desqueyroux-Faúndez, R. 1996. Antimalarial activity: the search for marine-derived natural products with selective antimalarial activity. Journal of Natural Products, 59: 710-716.

Vikas, D.K.; Vishweshwar, R.; Lakshmi, N.M. 2000. Current status of artemisinin and its derivatives as antimalarial drugs. Life Science, 66: 279-300.

Zani, C.L.; Chaves, P.P.G.; Queiroz, R.; Mendes, N.M.; Oliveira, A.B.; Cardoso, J.E.; Anjos, A.M.G.; Grandi, T.S. 1995. Brine shrimp lethality assay as a prescreening system for antiTrypanosoma cruzi activity. Phytomedicine, 2: 47-50.

Recebido em 21/05/2010

Aceito em 23/09/2010 